\title{
nature
}

5 May 2005 Volume 435 Issue no 7038

\section{In pursuit of systems}

The study of functioning groups of molecules is an important frontier of biology at reductionist and holistic levels. Central to the long-term goals of scientific research, it brings its own challenges of infrastructure and evaluation.

W hat is the difference between a live cat and a dead one? One scientific answer is 'systems biology'. A dead cat is a collection of its component parts. A live cat is the emergent behaviour of the system incorporating those parts. There is certainly a vast distance to go before we can fully encompass such a system within scientific description. So how is systems biology already moving us towards the fullest possible description of a live cat?

By focusing on the behaviour of individual proteins and other biomolecules, much of what gives life its unique properties can be missed. To a systems biologist, the network of interactions formed by these components is more important than the molecules themselves. Properties such as robustness and evolvability, essential characteristics of life, then emerge from the topology of biological networks, independent of the constituents from which they are built.

Such a holistic view may sound dangerously soft-edged. Far from it. Systems biology couples the acquisition of comprehensive, highdefinition data sets to the construction of quantitative models and computer simulations. Indeed, it is an explicit aim of both the Kyoto Encyclopedia of Genes and Genomes and the Alliance for Cellular Signaling to construct a fully functioning computer model of a cell.

The present state of experimental systems biology is both tantalizing and frustrating. To provide the level of detail required for us to know what is going on in a cell, microarray technologies will need to be faster and require smaller samples; ways to label and follow more biological molecules within a cell must be discovered; new spectroscopictools to non-invasively measure multiple metabolite levels will need to be developed, and so on. Nature is committed to publishing studies that push back the technological frontier of what it is possible to know about important biological systems.

But technical wizardry and large data sets are only part of the systems-biology approach - a system is not fully understood until a quantitative model can be built. The role of modelling in biological research is controversial and can spark heated debates. What is clear, though, is that the wealth of experimental data emerging from systems biology would be uninterpretable without detailed models against which they can be compared.Advances in modelling and simulation are thus noless important than data collection.

Every discipline generates community infrastructures, and systems biology is no exception. In the past five years, systems-biology institutes, departments and initiatives have been springing up across the globe. New journals have been launched, including The Institution of Electrical Engineers' Systems Biology and Nature Publishing Group's Molecular Systems Biology. The latter, an author-pays, online-only journal, is a joint venture with the European Molecular Biology Organization and went live last month.

The exchange of models between researchers is imperative, so a welcome development last month was the launch of BioModels (www.ebi.ac.uk/biomodels), a curated database for the deposition of biological models. BioModels has built on the success of Systems Biology Markup Language (SBML) in providing a format for the presentation of models, allowing them to be implemented on different software platforms. Nature journals and Molecular Systems Biology support submissions involving SBML.

It is hoped that BioModels will form the basis of a universally accepted repository that can do for systems biology what GenBank and the Protein Data Bank have done for genetics and structural biology. Nature applauds such efforts and will encourage authors of papers containing suitable models to contribute them to BioModels.

Systems biology presents an intellectual challenge to scientists and journal editors alike. Papers in this field document a highly multidisciplinary endeavour. Reviewers of such papers are very good at dissecting the aspects that fall within their sphere of expertise, but are less insightful beyond. So it falls to editors to weigh their frequently conflicting opinions in taking balanced and clear-sighted decisions. As a multidisciplinary journal, Nature welcomes the particular challenges that systems biology presents.

\section{Fear and rambling at NASA}

The US space agency needs an injection of rationality.

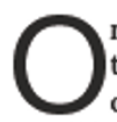
nly a confused space agency would consider shutting down the Voyager spacecraft as they approach the uncharted edge of the Solar System. Or cutting the basic research grants that provide the scientific basis for everything it does. Or cancelling satellites that make critical measurements of global climate change.

Last week a US National Academy of Sciences panel said that enough is enough, and called on NASA to reinstate some of its cancelled Earth-science projects (see page 9). NASA science chief AlDiaz denied that one of them, Glory, had even been cancelled. This highlights another disturbing trend: news of an impending cancellation is sent out or leaked, followed by a quick denial. Predictably, scientists reduced to chasing down rumours have turned fearful and angry.

NASA got into this mess through a variety of factors: an expensive initiative to send astronauts back to the Moon; uncertainty over how much the space shuttle will cost to repair; an accounting system that figures in all the agency's overhead costs for the first time; moves by Congress to claim hundreds of millions of dollars from NASA's budget; and unprecedented congressional permission tomove money between accounts. NASA managers who send out confused signals may not know themselves how much they can spend.

Into this muddle steps the new NASA administrator, Michael Griffin, who has already offered signs of hope. He, too, is impatient with the agency's accounting system and wants it fixed. He admits that not every project will survive NASA's change in direction, but at least wants decisions to be made rationally, meaning that the threatened Voyagers and the Hubble Space Telescope will get another hearing. The fiscal problems facing Griffin remain huge, but his willingness to address them honestly and directly are a good start. 